Revista 2020

\title{
La experiencia del riesgo de deserción como oportunidad y camino de formación en los estudiantes universitarios*
}

\author{
Floralba Barrero Rivera ${ }^{a}$
}

\begin{abstract}
Resumen: En este artículo se realiza un acercamiento teórico al concepto de deserción estudiantil universitaria, a partir de las narrativas experienciales del riesgo de deserción estudiantil (que surgen de las vivencias de los estudiantes), las cuales son analizadas y comprendidas desde su significado. Se propone explicar cómo estas narrativas pueden ser analizadas desde la hermenéutica y su dimensión ontológica, subjetiva e intersubjetiva. Lo anterior orienta el camino para convertir los escenarios de riesgo de deserción estudiantil en una propuesta de comprensión personal, que se traduzca en una búsqueda de la equidad educativa desde el desarrollo en lectura y escritura como fundamento de la propia comprensión de la experiencia de riesgo de deserción referenciada en el desarrollo de las potencialidades del estudiante como ser humano.
\end{abstract}

Palabras clave: riesgo de deserción; deserción universitaria; aprendizaje vivencial; educación universitaria.

Recibido: 1 de diciembre de 2019 Aceptado: 18 de mayo de 2020

Disponible en línea: 22 de octubre de 2020.

Cómo citar: Barrero, F. (2020). La experiencia del riesgo de deserción como oportunidad y camino de formación en los estudiantes universitarios. Academia y Virtualidad, 13(2), 57-68. https://doi. org/10.18359/ravi.4381

* Artículo de investigación.

a Doctora en Humanidades. Humanismo y Persona. Ecosocial Consultores, Bogotá, Colombia. Correo electrónico: fbarrero@ecosocialconsultores.com ORCID: http://orcid.org/0000-0002-1329-0401 


\title{
The drop-out risk experience as an opportunity and a learning path in university students
}

\begin{abstract}
This article provides a theoretical approach to the student attrition phenomenon based on students' drop out risk experience narrations (arising from the students' lives), which are analyzed and understood from their significance. It intends to explain how these narratives can be analyzed from hermeneutics and from their ontological, subjective, and intersubjective dimension. The foregoing guides the way to turn the student's dropout risk scenarios into a proposal for personal understanding, which translates into a search for educational equity from the development of reading and writing as a basis for one's own understanding of the dropping out risk experience referenced in the students' development of their potential as a human beings.
\end{abstract}

Keywords: drop-out risk; university attrition; experiential learning; university education.

\section{A experiência do risco de deserção como oportunidade e caminho de formação nos estudantes universitários}

Resumo: Neste artigo realiza-se uma aproximação teórica ao conceito de deserção estudantil universitária, a partir das narrativas experienciais do risco de deserção estudantil (que surgem das vivências dos estudantes), as quais são analisadas e compreendidas a partir de seu significado. Propõe-se explicar como essas narrativas podem ser analisadas a partir da hermenêtica e sua dimensão ontológica, subjetiva e intersubjetiva. Tudo isso orienta o caminho para transformar os cenários de risco de deserção estudantil numa proposta de entendimento pessoal, que se traduza numa busca da equidade educativa a partir do desenvolvimento em leitura e escrita como fundamento do próprio entendimento da experiência de risco de deserção referenciada no desenvolvimento das potencialidades do estudante como ser humano.

Palavras-chave: risco de deserção; deserção universitária; aprendizagem vivencial; educação universitária. 


\section{Introducción}

El objetivo de este artículo es evidenciar la relevancia que tienen las narrativas de los estudiantes que se encuentran en riesgo de deserción estudiantil. De esta forma, se muestra cómo su voz se convierte en texto, en imágenes y expresiones personales que reflejan escenarios de profunda necesidad de comprensión, así como nuevas interpretaciones de la realidad.

Es por esto por lo que se propone un acercamiento teórico que indica una ruta conceptual sobre cómo entender estas narrativas desde la hermenéutica, utilizándola como una perspectiva valiosa de comprensión de la realidad que enfrentan los estudiantes. En esta medida, la narrativa de la propia experiencia de riesgo de deserción de un estudiante se puede analizar desde tres dimensiones: ontológica, intersubjetiva y subjetiva.

Además de lo anterior, se propone que la experiencia de riesgo de deserción estudiantil universitaria, desde el campo de lo personal, lo académico, lo institucional y lo económico, puede ser conducida como una oportunidad para crear nuevos caminos de formación.

Para cumplir con ese objetivo principal, este artículo se divide en tres secciones: primero, se hará un recorrido teórico por las nociones de deserción estudiantil y de riesgo, desde el cuál se podrán evidenciar factores determinantes para identificar, posteriormente, su relación con el enfoque propuesto; se presentan los resultados de los significados de las narrativas de estudiantes en riesgo de deserción. Segundo, se hace un breve recuento de la categoría de formación y la de educabilidad en el referente de la experiencia que se inscribe en la comprensión del fenómeno desde la triple dimensión (ontológica, intersubjetiva y subjetiva), para tener el sustento teórico con el que se puede tratar el riesgo de deserción estudiantil. Tercero, se expone el camino que permitirá la formación de estudiantes a partir de ese riesgo de deserción, con el fin de lograr la equidad educativa.

\section{Riesgo de deserción estudiantil: una realidad diversa}

El concepto de riesgo se entiende, según Ulrich Beck (2000), como un estado perceptivo y de acción que se presenta de forma intermedia entre un estado inicial de confianza o de sensación de seguridad que se va diluyendo y una reacción medianamente calculada de anticipación y/o preparación para cualquier desastre que pueda venir conectado a esa pérdida de seguridad (Beck, 2000). Es por esto que estar en riesgo significa enfrentarse a situaciones que no se desean, situaciones que surgen como producto de la pérdida de estabilidad o confianza en algún proceso en el que se confiaba o del cual se creía que iba a continuar sin contratiempos. Una de estas percepciones de riesgo incorpora situaciones como los procesos educativos universitarios de un individuo. Concretamente, la ruta trazada entre el inicio de una carrera profesional y su culminación exitosa presenta riesgos sobre el futuro, en ámbitos como el económico, el personal, el ejercicio profesional y la vida familiar. En la medida en que estos riesgos se presentan pueden ser narrados y reconstruidos.

El riesgo como un escenario latente nos obliga a observar qué factores o realidades han permitido esa pérdida de seguridad y confianza en alguna realidad o proceso dado. La formulación teórica de Beck (2000) tiene una misión macro-sociológica, debido a que él buscaba un campo de análisis que le permitiese hablar de ciertos sucesos que afectaban la vida social del ser humano a escala global, asociados al riesgo de la confrontación atómica o de la redistribución de las lógicas y los procesos de la comunicación o la salud, determinados por la tecnología galopante producto de la Guerra Fría (Dingwall, 1999). Pero, al observarse en otras áreas del desarrollo de la vida humana moderna, como la educación superior en países latinoamericanos (Estrada, 2014), la noción de riesgo permite descubrir que existe otra gama de factores determinantes o influyentes en esa aparición y control del riesgo, 
y que, sin perder su relación con lo macro-sociológico, también son permeados por realidades y situaciones micro, que afectan al individuo en un nivel subjetivo e intersubjetivo.

Lo anterior permite descubrir que hablar de riesgo, ya sea de deserción estudiantil o de otro tipo, implica un análisis de diversos factores $y$, además, conlleva la observación de las diferentes dimensiones que afecten el riesgo (que permitan su aparición o su aumento). Por esto, el riesgo de deserción estudiantil se entiende como un fenómeno atravesado por múltiples causas, y desde su lectura teórica debe ser considerado como un fenómeno multidimensional (Barrero, 2016; Tinto, 2006).

Para el desarrollo de la argumentación es necesario presentar teóricamente una noción íntimamente relacionada con la deserción estudiantil, esta es la noción de fracaso o de fallo. Tinto (1993) explica que en los fenómenos de deserción estudiantil se suele responsabilizar al estudiante de manera directa por la interrupción de su proceso de formación; se le culpa de su "fracaso" estudiantil, como aquello que lo conduce a la deserción. Este autor logra llamar la atención sobre la responsabilidad que tienen las instituciones educativas en la aparición del riesgo de que un estudiante deserte; en otras palabras, cada caso de deserción estudiantil es también responsabilidad de las instituciones educativas (Tinto, 1993).

Se debe entender que "deserción estudiantil" y "riesgo de deserción estudiantil" no son lo mismo, así estén íntimamente relacionados entre sí. La deserción estudiantil se puede entender como la materialización de uno de los escenarios de riesgo. Esto permite entender que el riesgo de deserción estudiantil, y específicamente la experiencia de este riesgo, puede ser analizado y teorizado como un objeto de estudio particular.

Es importante recordar que las instituciones de educación superior tienen un papel vital respecto al riesgo de deserción estudiantil. Retomando a Beck (2000), debemos mencionar que la aparición y el aumento del riesgo se da, en gran parte, también como producto de una desatención del riesgo en sí mismo, o mejor, es producto de la desinformación sobre la posibilidad o presencia real del riesgo (Beck, 2000). Desde esta perspectiva resulta necesario analizar las dimensiones bajo las cuales se presenta el riesgo de deserción, ya que así es posible entenderlo de una manera objetiva para poder transformarlo en un camino de formación (Barrero, 2017).

El riesgo de deserción estudiantil responde a situaciones y realidades de carácter externo e interno para cada estudiante (Román, 2013). Román retoma esas situaciones que permiten la aparición del riesgo de deserción estudiantil desde la perspectiva del sistema educativo. Al referirse a factores endógenos, habla de factores interiores al propio sistema educativo -infraestructura escolar y planta profesoral, por ejemplo-, y al referirse a elementos exógenos considera elementos que van más allá del sistema educativo -presupuesto público, situación nutricional del estudiante o capital cultural de los estudiantes, por ejemplo-.

Aunque esta perspectiva ayuda a entender el entramado complejo de relaciones entre factores que pueden afectar el aumento o decrecimiento de riesgo de deserción estudiantil en determinadas situaciones o coyunturas, es una perspectiva restringida al punto de vista institucional. Esta mirada teórica hace del estudiante un sujeto de aplicación de acciones por parte de las instituciones educativas o del sistema educativo. Aquí, la propia experiencia del estudiante pasa a un plano de pasividad y receptividad. Se entiende que la institución o el sistema deben "lidiar" con, por ejemplo, el capital cultural del estudiante y, desde allí, realizar sus diagnósticos y plantear sus soluciones. Si bien defendemos la idea de responsabilizar a la institución educativa (a todas las instituciones educativas, para su efecto), es necesario hacer una primera reflexión, y también resolutiva, desde la propia experiencia de riesgo de deserción del estudiante, desde sus narrativas, desde sus dimensiones humanísticas (Barrero 2017).

Desde esta otra perspectiva, se busca rescatar el relato y la narrativa como fuente de comprensión 
de la experiencia de riesgo de deserción estudiantil. Utilizando metodologías de corte cualitativo, se pueden desarrollar diferentes exploraciones sobre aquellos factores que alteran el riesgo de deserción estudiantil. Diversos estudios han hecho análisis sobre este tema, explorando la relación entre distintos factores que conducen a la exclusión social, lo que genera ciertas lógicas de las relaciones de género y la deserción estudiantil de mujeres jóvenes, por ejemplo, de aquellas que se hayan en relaciones sentimentales en proceso de formalización (Estrada, 2015).

Pero de todos los factores que parecen incidir de manera directa en el aumento del riesgo de deserción estudiantil está el capital cultural (su ausencia o carencia). Este representa uno de los ítems más significativos, ya que plantea diversos retos y restricciones, especialmente ligados a su acceso a los códigos lingüísticos y a la falta de habilidades relacionadas con la lectura y la escritura (Barrero, 2017). En otras palabras, se pudo precisar como uno de los principales motivos de riesgo de deserción la carencia de capital cultural, concretamente la falta de habilidades para leer y escribir enfocadas en el aprender.

El capital cultural, así como su (desigual) distribución, el acceso (o la restricción) al mismo y su forma de generar distinción entre distintas personas y grupos sociales hacen parte de una realidad social y de unas mecánicas relacionales, es decir, están sujetos a las relaciones sociales -las estructurales y las interpersonales- (Bourdieu, 1987). El acceso a este capital, como a los demás (el social, económico y simbólico), es desigual, y los espacios en donde se adquiere este capital cultural, como las instituciones educativas, son reproductoras de estructuras sociales, específicamente, de desigualdad (Bourdieu, 1987).

Esto plantea que, si se lograse, desde una perspectiva relacional, combatir el acceso desigual al capital cultural, no solo se estaría combatiendo el riesgo de deserción estudiantil, convirtiéndolo en caminos de formación, sino que además se estaría realizando un esfuerzo por no permitir la reproducción de ciertas estructuras de distinción y desigualdad social (Barrero, 2017).

\section{Resultados de las narrativas de estudiantes en riesgo de deserción estudiantil}

A continuación, se resume los resultados del estudio (Barrero 2017), donde el enfoque narrativo fue el recurso de los estudiantes que voluntariamente expresaron su experiencia y que, desde sus narraciones, representan la acción de la vida cotidiana y de la vida en contextos especializados, en este caso la comunidad en riesgo de deserción estudiantil de la Universidad de San Buenaventura, sede Bogotá. El estudio se apoyó en la construcción de un corpus de narraciones y relatos autobiográficos de estudiantes en riesgo de deserción; en total el corpus cuenta con 120 narraciones de estudiantes que participaron voluntariamente. Este material fue codificado y sistematizado teniendo en cuenta la codificación abierta y axial.

Ahora bien, son dos los ejes que constituyen el análisis: el primero se concentra en la exposición de las categorías encontradas en la codificación a) capitales, b) sistema educativo y c) autobiografías, identidades y subjetividades. El segundo momento contiene las reflexiones sobre los significados atribuidos por los estudiantes al riesgo de deserción dando lugar al análisis sobre la deserción estudiantil.
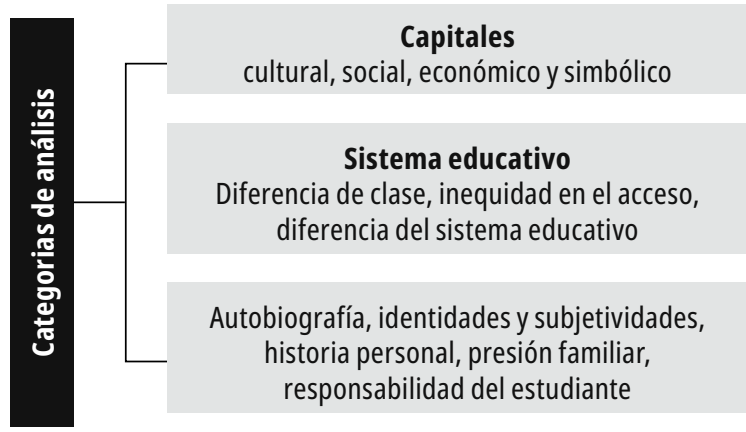

Figura 1. Categorías de análisis.

Fuente: elaboración propia.

\section{Primera categoría: capitales}

De acuerdo con Bourdieu, los capitales se definen como

los elementos que se disputan los miembros de un campo en las luchas permanentes que implican la 
construcción de dicho espacio social, estos toman tiempo para ser acumulados y su distribución y relevancia dan cuenta de la estructura inmanente del mundo social (Bourdieu, 1986, p. 46).

Los capitales se ponen en juego en campos particulares, como por ejemplo el campo universitario (Bourdieu, 1979, 1989, 2001). La posición desigual de estos capitales entre los individuos del campo (estudiantes, en el caso del estudio) implica la constitución de unas posiciones diferenciadas, que tienen un vínculo estrecho con la exclusión; esto porque no todos los estudiantes que luchan por la obtención del conocimiento disponen del capital suficiente para tener éxito en las acciones emprendidas, y esta carencia termina por excluirlos del campo.

En otras palabras, la posesión del capital, en cualquiera de sus formas, es un factor clave para determinar la posición y posible trayectoria de un estudiante en el campo universitario, esto es, determina la posibilidad de obtener éxito o fracasar en los proyectos universitarios que emprende. En esta categoría los resultados muestran que los estudiantes no poseen, en su gran mayoría, el capital cultural. Para la Institución de Educación Superior, es indispensable que los estudiantes posean o desarrollen habilidades ideales, como saber leer, escribir, analizar, investigar, destrezas necesarias para iniciar con éxito la vida universitaria. Sin embargo, en muchos casos no se cumplen estas condiciones, tal como lo verifica el estudio en mención. Seguido se encuentra el capital económico y simbólico. Lo mencionado contribuye a que los estudiantes tengan la sensación de exclusión del campo, motivo que conduce en muchos casos a la deserción estudiantil universitaria.

\section{Segunda categoría: sistema educativo}

Esta categoría se configuró con cuatro indicadores analíticos: 1) diferenciación por clase, 2) inequidad en el acceso, 3) desconexión entre la secundaria y la universidad y 4) diferencias entre educación rural y urbana. Los resultados de esta categoría muestran que los estudiantes reconocen los esfuerzos que realiza el sistema educativo por brindarles su apoyo; sin embargo, el sistema constantemente privilegia lo homogéneo, por lo que las trayectorias individuales son invisibilizadas. En consecuencia, las predicciones generales sobre fenómenos como la deserción estudiantil universitaria son orientadas por la normatividad de los reglamentos institucionales.

\section{Tercera categoría: autobiografía, identidades y subjetividades}

Esta categoría se configuró con tres indicadores analíticos: 1) historia personal, 2) presión familiar y 3) responsabilidad del estudiante. La expresión autobiográfica de la narración del estudiante constituye una capacidad fundamental de la especie humana y desempeña un papel significativo en la construcción de la experiencia.

Las voces de los estudiantes revelaron en sus resultados la importancia de las trayectorias individuales, sus anhelos y también sus dificultades expresadas a lo largo de sus narraciones. Sus voces clamaron al unísono la necesidad de ser escuchados, su autobiografía posee un valor propio que queda más allá del número para darle vida a una historia que reclama su presencia, entre otros que narran dificultades similares.

En conclusión, estas categorías permitieron configurar el diálogo entre el sustento teórico del estudio, la teoría de Pierre Bourdieu y lo que emergió de las narraciones de los estudiantes en riesgo de deserción (Barrero, 2017).

El significado de las narrativas de los estudiantes asociados al riesgo de deserción estudiantil permitió la construcción teórica desde la perspectiva de los estudiantes, los significados que cobra dicha experiencia de riesgo y, por lo tanto, contribuye a comprender el fenómeno desde una perspectiva integradora, a la elaboración de una teoría sustantiva sobre la deserción estudiantil universitaria. Aquí son las múltiples voces de estudiantes en riesgo de deserción las que permiten desarrollar las interpretaciones conceptuales; son las voces de los estudiantes las que narran sus experiencias y sentimientos con respecto a su propio riesgo de deserción. 
Los significados de las distintas narraciones permitieron crear teoría, con nuevas reflexiones sobre la necesidad de abordar el fenómeno de la deserción desde una mirada social y de concebirlo como un problema de equidad educativa que deben asumir las humanidades. Ello, como el resultado de la intersección de categorías sociales que convergen en la noción de equidad educativa como oportunidad de permanencia. Las voces de los estudiantes le confieren significado a lo social, institucional y subjetivo, lo cual lleva a la conclusión de que la deserción estudiantil es producto de diversos aspectos que se intersectan, con una unidad de análisis común, que no es otra cosa que la diferencia de volúmenes de capital cultural.

Uno de los patrones recurrentes es la dificultad en la lectura y la escritura, fuente permanente de profunda inequidad social y, como consecuencia, una vez más se ratifican los bajos volúmenes de capital cultural que traen los estudiantes una vez ingresan a las instituciones de Educación Superior.

\section{Formación y educabilidad como realidad antropológica contra el riesgo de deserción}

Desde el enfoque teórico de este artículo, la experiencia de los estudiantes en riesgo de deserción, que se encuentra en las narrativas, se entrelaza con la realidad de la formación y la educabilidad. Es más, la experiencia de un estudiante en riesgo de deserción, tomada como un fenómeno relacionado con la propia vida y contexto del estudiante, permiten convertir el análisis de esa vivencia en un camino de formación.

En diferentes discusiones teóricas, se ha argumentado que aprender es una función inherente y paralela a la vida del ser humano. En otras palabras, se ha aceptado que el ser humano "vive mientras aprende" o "vive aprendiendo". Desde este punto de vista, "aprender", como verbo, refleja una relación con el conocimiento que parece lineal o unidireccional, en la que el ser humano adquiere conocimientos, pero esta relación es mucho más profunda. Es por esto que el concepto de formación nos permite acercarnos más a la relación entre conocimiento y ser humano.

Bajo una mirada centrada en la formación universitaria, el concepto de "formación" aparece como un proceso a través del cual una persona se abre camino hacia los conocimientos de realidades específicas y desarrolla maneras de estudiarlas (Ruiz, 2002). Este concepto trae implícita la noción de "apropiación", lo cual revela que en los procesos de formación existe una agencia, un ordenamiento encauzado de acciones que vienen tanto por parte de los educadores o quienes guían el proceso de formación como de quienes se educan o son sujetos de formación. El componente relacional está siempre presente en el proceso de formación, y denota una agencia humana que se da entre personas (formador y formando) y entre personas y conocimiento.

Según el concepto de formación universitaria, mencionado anteriormente, el proceso va enfocado hacia la absorción y comprensión de la realidad, pero la formación en todos sus aspectos tiene un objetivo mucho más compenetrado con el ser humano. Desde una perspectiva antropológica, el ser humano es un ser/animal inacabado y que tiende a no completarse, en el sentido de un aprendizaje y un proceso de construcción constante, mediado por sus realidades sociales, psicológicas, etc. Así, el concepto de formación tiene también una relación con el hecho de "dar forma", no como se da forma a un material (no es "moldear"), sino de brindar estructura, de "estructurar" (Vanegas, 2004).

Para Gadamer, la formación como parte de la educación del ser humano encierra un proceso íntimo, es decir, la persona que se forma y se educa se está desarrollando a sí misma, "accede" a sí misma (Fernández-Nieto, 2016; Gadamer, 2000). La formación es un proceso que depende de la comprensión de la vida y la experiencia del ser humano, pero que también lo forma como producto de ese proceso. Así, un proceso de formación (y como se verá en un instante, también bajo el concepto de educabilidad) tiene que hacerse cargo también de aquellas dimensiones que comprenden al ser humano, desde lo social, lo psicológico, lo 
biológico, lo cultural y demás dimensiones, hasta la moral y la ética (Barrio, 2007). Atender las experiencias de riesgo de deserción desde esta perspectiva, que toma en cuenta la formación del ser humano en todos esos aspectos, es la clave para transformar esas experiencias de riesgo y llenarlas de significado para superarlas.

En todo caso, el concepto de formación, como se propone en este artículo, se entiende como un proceso que va más allá de lo educativo, y que se inscribe en un proceso más trascendental de transformación de la misma esencia (estructuras) del ser humano (Villegas, 2008); lo cual también lo convierte en un problema antropológico (Fernández-Nieto, 2016). La formación, entonces, no es algo que solo compete al escenario de la educación (universitaria, en este caso), sino que involucra también el proceso de crecimiento y formación del ser humano en otras dimensiones de su vida (Villegas, 2008). Pensar el riesgo de deserción como una posibilidad de construcción de un camino de formación involucra, específicamente, su componente relacional y antropológico. Atender el riesgo de deserción implicaría atender la realidad sociocultural y lingüística del estudiante para combatir aquel riesgo $y$, del mismo modo, convertirlo en una oportunidad para continuar la formación.

Para poder hacer el vínculo entre el escenario de la educación superior y las experiencias de riesgo de deserción como posibles caminos de formación, es necesario también retomar el concepto de educabilidad. En un principio, este concepto versaba únicamente sobre la capacidad de plasticidad de los educandos de ir acoplándose a nuevas experiencias y nuevos conocimientos (exposición a la realidad). Desafortunadamente, esa noción primera de educabilidad supone ver a los educandos como "objetos" de enseñanza, como materias primas sobre las cuales se imprimen o se construyen ciertas matrices de conocimiento (Gallego y Pérez, 1999). Contrario a esto último, la educabilidad se refiere más a un proceso no solo de adaptación, sino de interacción, y no solo entre individuo y entorno o entre individuo y "otros", sino más bien entre el individuo y sí mismo y entre el individuo y su realidad (García, 1993). De allí se desprende la relación con la trilogía dimensional de la comprensión y la narrativa (que se expondrá en el apartado siguiente).

La educabilidad resalta un componente relacional, en el que el concepto ya no solo hace referencia a una "capacidad" que tiene el educando de ser educado, sino que adquiere agencia a través de las interacciones con otros, por lo cual depende profundamente del reconocimiento del otro, de la otredad. Esto significa que la educabilidad se transforma también en, y depende de, que el individuo ejerza su agencia en cuanto a que adquiera la determinación de "hacerse educable" como una condición ontológica (Gallego y Pérez, 1999). Pero, así como la educabilidad (desde donde se podría afirmar que alguien tiene mayor o menor pretensión o decisión de ser educable, de ser un educando) es relacional en cuanto a su construcción, también significa que a partir de las relaciones interpersonales, de la dimensión social y cultural, del acceso al capital cultural o de otras características que componen la realidad se puede llegar a escenarios en donde los individuos no tengan la oportunidad de desarrollarse como educandos, es decir, en donde disminuya su educabilidad. Es por este doble componente de la educabilidad que, al intervenir las experiencias de riesgo de deserción estudiantil, estas se pueden transformar en caminos de formación, en otras palabras, se afecta de manera positiva la educabilidad.

\section{Comprensión y narrativa: tres dimensiones}

El ejercicio teórico del riesgo de deserción estudiantil que se propone en este artículo está directamente relacionado con la comprensión de las experiencias de riesgo de deserción estudiantil. Una comprensión de las narrativas de experiencia del riesgo de deserción estudiantil, específicamente. Para esto, es necesario explorar teóricamente la comprensión, desde un punto de vista teórico filosófico que nos dé las herramientas para abordar el tema del riesgo de deserción estudiantil. Se hará desde una breve exploración de la hermenéutica de Gadamer y el concepto de comprensión. Según Gadamer, "la hermenéutica no significa tanto un procedimiento cuanto la actitud del ser humano 
que quiere entender a otro o que como oyente o lector quiere entender una manifestación verbal, siempre es, pues entender a un ser humano" (1999, p. 149).

La relación entre comprender y experiencia, entre comprender y vivir es tan íntima, asegura Gadamer, que básicamente los seres humanos vivimos comprendiendo, es decir, nuestra propia actividad humana está relacionada con la acción hermenéutica de ir comprendiendo (Gadamer, 1993). Comprender y vivir, así como comprender y la experiencia humana son realidades y actividades consustanciales (Ruiz, 2002).

Todo proceso de comprensión, entonces, lleva consigo una relación con el ser, pero también con un "estar en el mundo", y al mismo tiempo con un "ser en el mundo". Esta triple dimensión es la que guía la forma en la que podemos observar la comprensión, que es al mismo tiempo una reflexión sobre la experiencia. Las tres dimensiones de la experiencia humana y de la comprensión son la dimensión ontológica, la dimensión intersubjetiva y la dimensión subjetiva.

\section{Dimensión ontológica: el ser y la realidad}

La dimensión ontológica de la comprensión y de la experiencia humana encierra la relación entre el ser (que en nuestro objeto de estudio sería el estudiante en riesgo de deserción) y el mundo. El mundo se entiende acá como el conjunto habitado y experimentado del ser humano, con el que se relaciona permanentemente y del que se alimenta (o se guía) para sus acciones (Ruiz, 2002) ${ }^{11}$.

El análisis sobre el ser, entonces, requiere la reflexión también sobre el mundo. La dimensión ontológica de la comprensión permite observar al ser desde un plano relacional, pero no de este ser en su relación consigo mismo (estar en el mundo, subjetivamente) ni de su relación con los demás (ser en el mundo, inter-subjetivamente), sino desde el plano mismo de habitar y actuar en el marco del mundo. En otras palabras, la dimensión ontológica es la forma de definirse, referirse y referenciarse en

1 No se correlaciona necesariamente con la noción de mundo de la física. el mundo. Comprender la dimensión ontológica es conocer la relación entre el ser y la realidad, lo que ayuda a evidenciar los parámetros de vivencia (experiencia) y convivencia, ya que la dimensión ontológica, aunque no hace referencia a la dimensión intersubjetiva, sí hace referencia a una "postura" en el mundo. Esa posición y su comprensión son la clave inicial para convertir, por ejemplo, una experiencia de riesgo de deserción estudiantil en un camino de formación.

\section{Dimensión intersubjetiva}

Los seres humanos experimentan su ser en el mundo (su relación con la realidad) gracias a su actividad lingüística, intelectiva y selectiva, lo que significa que el ser en el mundo es el resultado de actuar "en relación con" y "pensar con relación a", es decir, es una actividad en conjunto (Ruiz, 2002). Esto configura las características de la interacción social de los estudiantes, en lo referente al componente relacional de la comprensión y la experiencia humana.

Para clarificar la dimensión intersubjetiva es importante mencionar que esa realidad, ese marco de acción, parámetro de existencia, solo es posible gracias a la interacción. De forma simultánea, no puede existir una realidad, un mundo, un marco de referencia sin unas relaciones inter-subjetivas constantes que estén construyendo, expandiendo o rompiendo los límites mismos de la realidad. Esto nos permite imprimirle un carácter social a la realidad, de tal forma que el mundo es también una construcción social, no solo intelectual ni física (Ruiz, 2002).

Para Pierre Bourdieu (1989), la realidad social es un continuum histórico irreductible a una visión equilibrada y mecanicista que reduzca e invisibilice la experiencia de los agentes que lo componen. En palabras del autor:

se puede así representar al mundo social bajo la forma de un espacio (con muchas dimensiones) construido sobre la base de principios de diferenciación o de distribución constituidas por el conjunto de las propiedades activas dentro del universo social que es considerado, es decir, capaces de conferir a su detentador la fuerza, el poder en ese universo. Los 
agentes y los grupos de agentes son definidos de este modo por sus posiciones relativas en ese espacio. Cada uno de ellos está acantonado en una posición o en una clase precisa de posiciones vecinas (por ejemplo, en una región determinada del espacio) y no se puede realmente -aun si puede hacerse en el pensamiento- ocupar dos regiones opuestas del mismo espacio (Bourdieu, 1989, p. 28).

\section{Dimensión subjetiva}

Comprendiendo el mundo y comprendiendo a los otros, entendiéndolos y entendiéndose con ellos, el ser humano se comprende a sí mismo, se cultiva y cultiva su vida interior, potencia sus proyectos vitales. En esta dimensión radica el tema de la identidad y de la integridad del ser humano.

Esta dimensión tiene como característica fundamental la reflexividad, el pensamiento crítico y analítico como forma de comprender al mundo. "Estar en el mundo" es la dimensión a través de la cual, junto con la relación con la realidad y con otros sujetos, se completa y se complementa la comprensión y la experiencia humana.

Estas dimensiones de la comprensión permitirían que los estudiantes en riesgo de deserción encuentren un camino de formación desde sus propias dificultades, hallando el sentido de la lectura y escritura como posibilidad de enfrentar el riesgo no solo de su vida académica, sino de su realidad. Finalmente, abren la posibilidad de comprender que su experiencia en la educación no solo es un proceso pedagógico y de formación, sino que es también un proceso de ser y estar en el mundo, de construcción subjetiva.

\section{Perspectiva hermenéutica (intersubjetiva, ontológica y subjetiva) en relación con el riesgo de deserción}

Es posible entender que el rasgo principal de la inteligencia humana tiene que ver con la comprensión. De esta manera, los estudiantes en riesgo de deserción pueden comprender que, desde la experiencia narrada, donde se reflejan las dificultades que tienen con respeto a la lectura y escritura, se crea una posibilidad de comprensión para acercarse de otra manera a la carencia de habilidades relacionadas con lectura y escritura que en un momento pudo ser motivo de riesgo de deserción.

Con el fin de articular la experiencia de riesgo de deserción como camino de formación en los estudiantes, es necesario partir de la afirmación de Gadamer cuando plantea que la "comprensión es el modo de ser de la vida humana en cuanto que es poder ser y posibilidad" (Citado en Ruiz, 2002, p. 74). La comprensión es apertura y desarrollo de posibilidades. Si el estudiante puede llegar a comprender su riego de deserción es preciso permitirle la oportunidad de llegar a ser de otra manera, de vincularse en primera instancia con la lectura y la escritura desde sus potencialidades y reflexionar sobre su ser $\mathrm{y}$ sus posibilidades.

Como se mencionó anteriormente, es necesario afirmar que vivimos comprendiendo. En este sentido, se entiende que no solo se involucra la actividad de las facultades intelectuales, sino también el complejo sistema mediante el cual percibimos y nos relacionamos con el mundo -la naturaleza, las cosas, los artefactos y los otros-. De ahí la importancia de trabajar con los estudiantes desde su historia personal los factores de riesgo de deserción, especialmente la lectura y la escritura, y generar procesos de comprensión. Es necesario valorar y ofrecer el proceso de comprensión de la experiencia en riesgo de deserción estudiantil.

Reconocer la unidad interna de la inteligencia de los sentidos, más allá de la oposición tradicional entre la razón y la sensibilidad, ofrece un camino de formación para los estudiantes en riesgo de deserción. Entonces, es esencial desarrollar la capacidad lingüística a partir de la profundización de la lectura y escritura en el contexto de las habilidades comunicativas. De esto se desprende que la capacidad lingüística será aquella que es vivida desde la experiencia de la lectura y escritura, lo que permitirá que los estudiantes encuentren el camino de formación para posibilitar una educación centrada en sus potencialidades.

En esta era de la racionalidad técnica, la era digital, la era de las telecomunicaciones o era de la información, se ha perdido la inteligencia de los sentidos, se acentúa la incapacidad para escuchar y 
falta de sentido histórico; por esto es conveniente y urgente desarrollar la comprensión (Ruiz, 2002).

El estudiante en riesgo de deserción debe identificar su dimensión ontológica y entender el lugar que ocupa en la universidad. Se deben plantear preguntas sobre cómo es su mundo como estudiante en riesgo de deserción. Este ejercicio de comprender, para el estudiante, es vital para construir un camino de formación: vivir es estar con otros. El estudiante comprendería su riesgo de deserción en un ambiente concreto que le invita a reflexionar sobre el mundo en que está.

Comprender este modo de ser es la tarea de la formación en las humanidades de la pedagogía desde la reflexión antropológica del ser humano (Ruiz, 2002). La primera reflexión del estudiante en riesgo de deserción corresponde al cuidado de estar en ese mundo de riesgo. Esto lo induce a desarrollar modos de acción y de saber vivir que permitan el equilibrio de las fuerzas que nos hacen existir de una manera razonable.

Los estudiantes en riesgo de deserción deben interactuar con otros estudiantes que padezcan el mismo riesgo. El acercamiento debe ayudar a encontrar el sentido de estar en la universidad y responder a qué han venido a la universidad. El superar la dificultad para aprender a leer de manera comprensiva le ofrece un camino de posibilidad para saber estar en el mundo.

Las bases de esta dimensión intersubjetiva son: el diálogo, la interdisciplinaridad y la acción comunicativa. Este ejercicio intersubjetivo que realizarían los estudiantes en riesgo de deserción desde el acercamiento a la lectura y escritura dejaría claro el cuidado por la lectura, por la capacidad y potencia que ofrece desarrollar estas habilidades comunicativas.

\section{La corresponsabilidad de la permanencia en los procesos educativos: ¿un camino hacia la equidad educativa?}

Ofrecer a los estudiantes la posibilidad de potenciar sus experiencias en riesgo de deserción permitiría aumentar sus volúmenes de capital cultural en el caso concreto de adquirir códigos lingüísticos que los acerquen a la lectura y la escritura, para así valorar su importancia en una sociedad de permanente riesgo. Acercarse a la experiencia de la lectura en la perspectiva de las tres dimensiones de la comprensión ofrecería grandes presupuestos para formular las políticas de equidad educativa.

Desde esta perspectiva, en la que las tres dimensiones de la comprensión ayudan a evidenciar las experiencias de riesgo de deserción estudiantil, se da un rol muy importante al estudiante como parte del objeto de estudio. ¿Cómo lograr que tenga acceso a todas las reflexiones que lo conduzcan a caminos de formación desde la comprensión de sus diferentes riesgos (académico, sociodemográfico, institucional y personal)? Es por eso por lo que, desde esta posibilidad teórica, se hace un llamado a las instituciones educativas para que participen de forma activa, pero dando el protagonismo al estudiante y a su experiencia de riesgo de deserción estudiantil (su riesgo de "fracaso" en el mundo estudiantil, de no ser en su proceso de formación), todo con el objetivo de poder promulgar un acceso más equitativo al capital cultural y al desarrollo de la lectura y la escritura como fuente de equidad. Desde ese presupuesto teórico, se podría combatir el riesgo de deserción estudiantil, influyendo significativamente en la disminución de los niveles de deserción estudiantil.

\section{Referencias}

Barrero, F. (2016). Investigación en deserción estudiantil universitaria: educación, cultura y significados. Revista de Educación y Desarrollo Social, 9(2), 86-101. DOI: https://doi.org/10.18359/reds.948

Barrero, F. (2017). Significados de la deserción estudiantil universitaria construidos a partir de las experiencias y las narraciones de estudiantes en riesgo de deserción (Tesis doctoral). Bogotá: Universidad San Buenaventura.

Barrio, J. M. (2007). Dimensiones del crecimiento humano. Educación y Educadores, 10(1), 117-134.

Beck, U. (2000). Retorno a la teoría de la "Sociedad del riesgo”. Boletín de la Asociación de Geógrafos Españoles, (30), 9-20. 
Bourdieu, P. (1986). The forms of capital. En J Richardson, Handbook of theory and research for the sociology of education. New York: Greenwood.

Bourdieu, P. (1987). Los tres estados del capital cultural. Sociológica, 5, 11-17.

Bourdieu, P. (1989). El espacio social y la génesis de las “clases". Estudios sobre las Culturas Contemporáneas, 3(7), 27-55.

Bourdieu, P. (2001). Las formas de capital (trad. M José Bernuz Benítez ). En poder derecho y clases sociales (pp 131-164)(2da ed.) Bilbao:Desclee de Brouwer.

Dingwall, R. (1999). "Risk Society": The Cult of Theory and the Millennium? Social Policy \& Administration, 33(4), 474-491. DoI: https://doi.org/10.1111/1467-9515.00164

Estrada, M. (2014). Afiliación juvenil y desafiliación institucional. El entramado complejo de la deserción en la educación media. Revista Mexicana de Investigación Educativa, 19(61), 431-453.

Estrada, M. (2015). Las jóvenes que desertan de la educación media: virajes y puntos de no retorno. Revista Latinoamericana de Ciencias Sociales, 13(2), 995-1008. DoI: https://doi.org/10.11600/1692715x.13231190214

Fernández-Nieto, J. B. (2016). Antropología pedagógica, fin para la educación. Revista Escuela Abierta, 19, 4963. DoI: https://doi.org/10.29257/EA19.2016.04

Gadamer, H. (1993). Verdad y Método. Salamanca: Sígueme.

Gadamer, H. (2000). La educación es educarse. Barcelona: Paidós.
Gallego, R. y Pérez, R. (1999). Aprendibilidad, enseñabilidad y educabilidad en las ciencias experimentales. Revista Educación y Pedagogía, 6(25), 87-117.

García, J. (1993). El concepto de educabilidad y el proceso educacional. Teoría de la Educación, 5, 11-32.

Román, M. (2013). Factores asociados al abandono y la deserción escolar en América Latina: una mirada en conjunto. Revista Iberoamericana Sobre Calidad, Eficacia y Cambio en Educación, 11(2), 33-59.

Ruiz, M. Á. (2002). Las humanidades como experiencia de comprensión en la formación universitaria. Revista Educación y Pedagogía, 14(34), 69-83.

Tinto, V. (1993). Leaving College: Rethinking the Causes and Cures of Student Attrition. Chicago: University of Chicago Press. DoI: https://doi.org/10.7208/chicago/9780226922461.001.0001

Tinto, V. (2006). Research and Practice of Student Retention: What Next? Journal of College Student Retention, 8(1), 1-19. DoI: https://doi.org/10.2190/4YNU-4TMB22DJ-AN4W

Vanegas, M. E. (2004). El concepto pedagógico "Formación” en el universo semántico de la educación. Revista Educación, 28(2), 13-28. DoI: https://doi.org/10.15517/ revedu.v28i2.2251

Villegas, L. A. (2008). Formación: apuntes para su comprensión en la docencia universitaria. Revista de currículum y formación del profesorado, 12, 1-14. 